\title{
Structure and mechanism of the RNA dependent RNase Cas13a from Rhodobacter capsulatus
}

\author{
Kick, L.M., von Wrisberg, M.-K., Runtsch, L. S. and Schneider, S.*
}

Ludwig-Maximilians University, Department of Chemistry Butenandtstr. 5-13, 81377 Munich, Germany

E-mail: sabine.schneider@cup.Imu.de

\begin{abstract}
Cas13a are single-molecule effectors of the Class II, Type VI family of CRISPR-Cas systems that are part of the bacterial and archaeal defense systems. These RNA-guided and RNA-activated RNA endonucleases are characterized by their ability to cleave target RNAs complementary to the crRNAspacer sequence, as well as bystander RNAs in a sequence-unspecific manner. Due to cleavage of cellular transcripts they induce dormancy in the host cell and thus protect the bacterial population by aborting the infectious cycle of RNA-phages. Here we report the structural and functional characterization of a Cas13a enzyme from the photo-auxotrophic purple bacteria Rhodobacter capsulatus. The X-ray crystal structure of the RcCas13a-crRNA complex reveals its distinct crRNA recognition mode as well as the enzyme in its contracted, pre-activation conformation. Using sitedirected mutagenesis in combination with mass spectrometry, we identified key-residues responsible for pre-crRNA processing by RcCas13a in its distinct catalytic site, and elucidated the acid-base mediated cleavage reaction mechanism. In addition, RcCas13a cleaves target-RNA as well as bystander-RNAs in Escherichia coli which requires its catalytic active HEPN (higher eukaryotes and prokaryotes nucleotide binding) domain nuclease activity. Our data provide further insights into the molecular mechanisms and function of this intriguing family of RNA-dependent RNA endonucleases that are already employed as efficient tools for RNA detection and regulation of gene expression.
\end{abstract}

\section{Introduction}

Type VI CRISPR-Cas systems are characterized by their large single-protein effector nuclease with a composite active site consisting of two highly divergent Higher Eukaryotes and Prokaryotes Nucleotide-binding (HEPN) domains. The type VI CRISPR-Cas systems most likely evolved from toxin-antitoxin systems through insertion of a HEPN-encoding element/gene next to a CRISPR array, duplication of HEPN domain and multiple insertions that increased specificity of crRNA binding as well as multiple independent acquisitions of adaptation module (1). These systems can be found in a number of bacterial species with Cas13a (formerly C2c2) as the hallmark enzyme (1), but share an overall low sequence identity of $20-30 \%$. (2) Cas13a possess two distinct active sites, one for precrRNA processing and one for target-RNA cleavage. $(3,4)$ They become activated by sequence specific binding of a single stranded target RNA to the crRNA, which induces a conformational change in the composite HEPN domains, resulting in cleavage of the target ssRNA (cis-cleavage) and sequence-unspecific bystander ssRNAs (trans-cleavage) through its HEPN-nuclease site (3-8), which has been exploited for the in situ detection of viral RNAs. (9) In addition, in Listeria it was shown that activation of Cas13a leads to trans-cleavage of mRNA transcripts, that in turn leads to induction of dormancy in the host, which is sufficient to abort lytic infection and phage propagation in a cellular population.(10) This resembles the strategy of abortive infection systems where dormant enzymes are activated upon phage replication, resulting in inactivation of the cellular translation or transcription machinery, protein phosphorylation or alteration of the membrane integrity, leading to growth arrest or cell death, thus arresting phage infection and spreading. (11) Moreover, phages with target mutations that evade DNA-targeting by CRISPR-Cas systems are neutralized by Cas13a when 
activated by wild type phages. (12-15) Therefore, CRISPR-Cas13a systems prevent outbreaks of CRISPR-resistant phages by acting on the host and not directly on virus. (10)

Here we provide data on the structure and mechanisms of the Cas13a-endonuclease from the photoauxotrophic bacteria Rhodobacter capsulatus (RcCas13a), which shares $~ 20-25 \%$ sequence identity with the to date structurally characterized homologous enzymes from Lachnospiraceae bacterium (Lba) (8), Leptotrichia buccalis (Lbu) (7), L. shaii (Lsh),(4) and Listeria seeligeri (Lse). (10) The X-ray crystal structure of RcCas13a in complex with its crRNA elucidates how RcCas13a specifically recognizes its crRNA and shows the enzyme in a contracted, pre-activation conformation. In order to determine amino acid residues responsible for pre-crRNA processing by RcCas13a in the not conserved likely processing catalytic site, we employed site-directed mutagenesis in combination with in vitro activity assays and mass spectrometry. This allowed us not only to identify key-residues responsible for pre-crRNA processing by RcCas13a, but also to elucidate the reaction mechanism. Moreover, we could show that RcCas13a can be activated in Escherichia coli, where it cleaves targetRNA as well as bystander-RNAs, which requires a catalytic active HEPN (higher eukaryotes and prokaryotes nucleotide binding) domain. In summary, this study provides further insight into the molecular mechanisms of this intriguing family of RNA-dependent RNA endonucleases.

\section{Results and Discussion}

\section{Overall structure of the RcCas13a-crRNA binary complex}

The reference genome of $R$. capsulatus contains three CRISPR-Cas systems: Two Class I systems (Type I and Type III) as well as one Class II, Type VI system (Supporting Fig. S1), with the Cas13asignature sequence encoding a $145 \mathrm{kDa}$ protein sharing the family-typical HEPN-endonuclease domains, with the conserved Arg-His-Arg-His catalytic tetrad (Fig. 1A and Supporting Fig. S2). (16) We expressed RcCas13a alone and together with a CRISPR-locus in E. coli and purified apoRcCas13a as well as the enzyme in complex with the 58nt crRNA (RcCas13a-crRNA binary complex). The RcCas $13 a-c r R N A$ binary complex exhibits a markedly increased stability of about $10^{\circ} \mathrm{C}$ (Supporting Fig. S3+4). We determined the X-ray crystal of the binary complex by experimental phasing, using crystals of SeMet labelled RcCas13a protein and refined the final coordinates against data collected on native RcCas13a-crRNA complex crystals to $2.1 \AA$ resolution. (For X-ray data processing, structure solution and refinement statistics see Supporting Table S1) Protein residues as well as nucleobases of the repeat-region and the first 16nt's of the spacer in the crRNA are well defined in the electron density. However, some nucleobases in the middle of the spacer sequence and solvent-exposed loop regions in the protein are flexible and partly disordered (Supporting Fig. 5). RcCas 13 a shares the common domain architecture and predominantly $\alpha$-helical folding topology of the Cas13a-family. (Fig. 1A+B and Supporting Fig. S6) However, we did not observe electron density for the C-terminal 120 aa residues, that are unique to RcCas13a. (Supporting Fig. S2) Using HHPRED (17) we recognized that the CTD has similarity to members of the cold shock domain-protein family, which are multifunctional RNA/DNA binding proteins, exhibiting a $\beta$-barrel fold. (18) (Supporting Fig. S7). Albeit the sequence identity is low ( 15\%), it is likely that the RcCas13a-CTD adopts a similar overall fold. Nevertheless, all our attempts to express and purify solely the RcCas $13 a-$ CTD domain for structural characterization failed (data not shown).

The binary complex-structure of RcCas13a shows the enzyme in a contracted, inactive conformation. Here, in the composite active site made up by the HEPN1 and HEPN2 domains, His 469 of the conserved catalytic tetrad (Arg 464+His 469/Arg 1052+His 1057) is shifted about $18 \AA$ away, preventing endonuclease activity prior to target RNA binding (Fig. 1B+C). Moreover, as common for Cas13a-endonucleases, it possesses two distinct active sites for crRNA processing and target RNA 
cleavage (Fig. 1C+D). This is in agreement with functional and structural studies on the homologs with $\sim 23 \%$ sequence identity to RcCas13a which demonstrated that target RNA binding to the binary complex induces a conformational change and rotation of the helical 2 ( $\mathrm{Hel} 2$ ) domain relative to the HEPN1 domain, resulting in rearrangement of the catalytic tetrad thereby switching-on the RNase activity (Supporting Fig S8) (4,7,8,10). Thus, the stable contracted conformation of the binary complex as observed in RcCas13a, likely prevents premature activation of the RNase function.
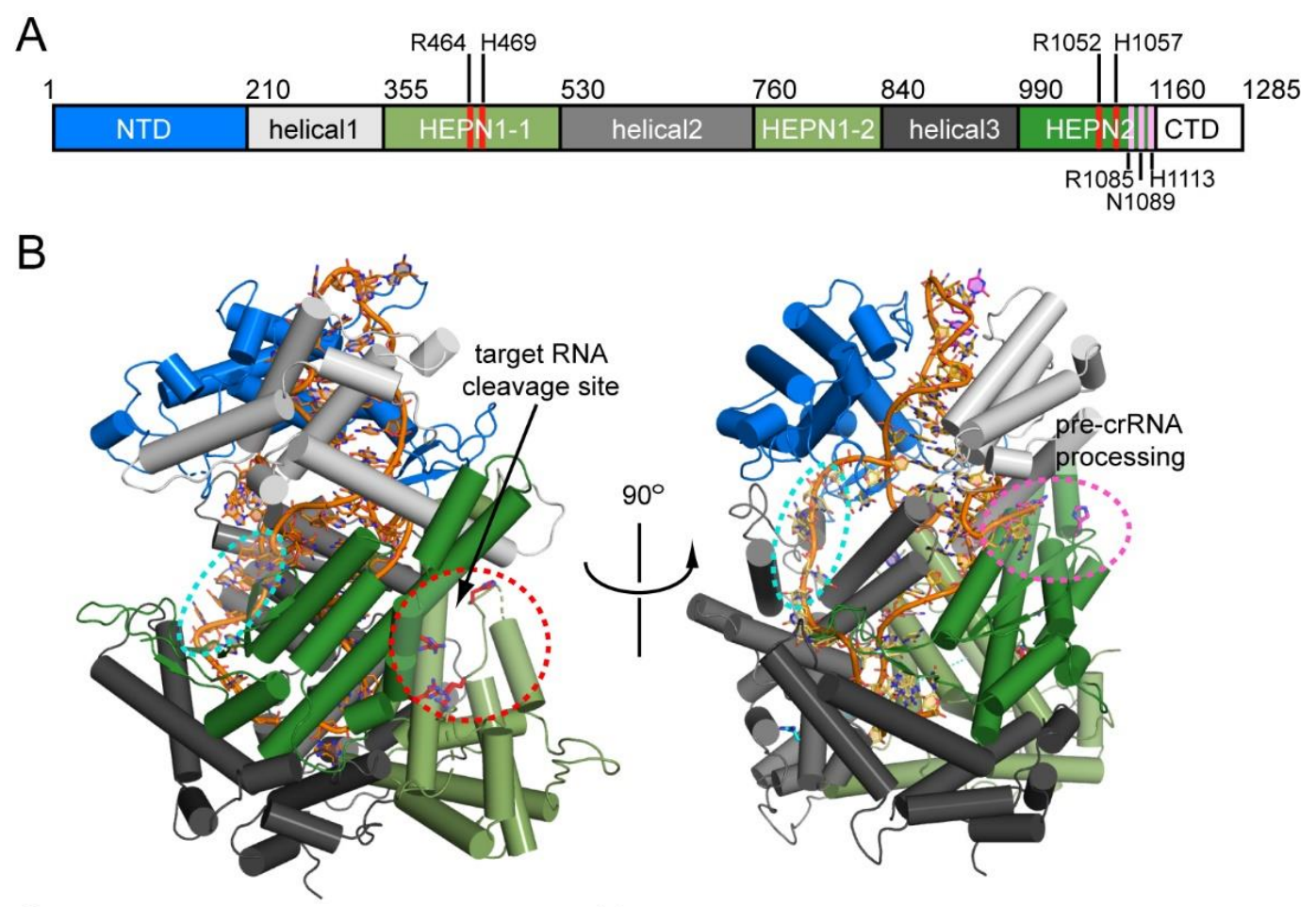

C
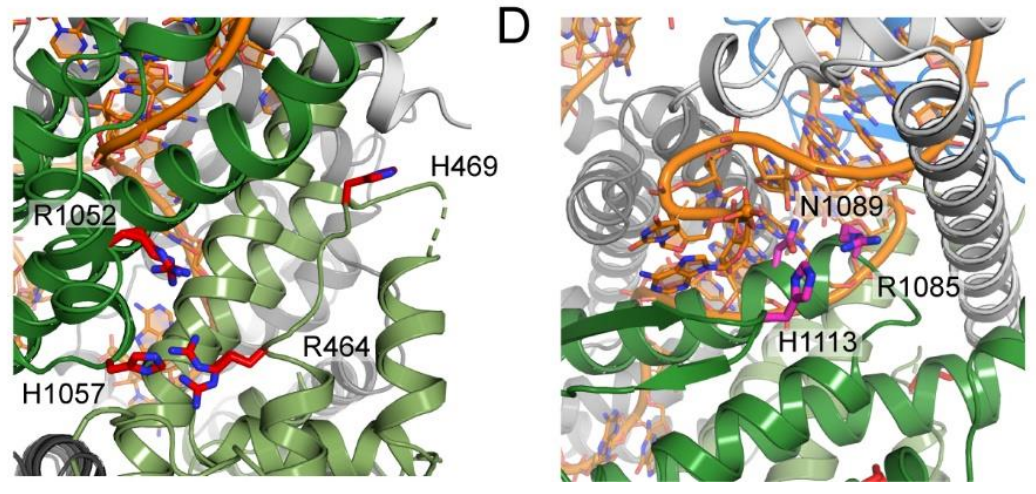

Fig. 1. Overall structure of the RcCas13a-crRNA complex. A) Domain-structure of RcCas13a with the residues important for the catalytic activity highlighted in red (trans-ssRNA cleavage) and pink (pre-crRNA processing), respectively. B) The location of the two independent active sites are indicated by a red circle, the seed region of the crRNA (position 10-14) by a cyan circle. C) Zoom in the RNase active site, with the residues crucial for catalytic activity (Arg 464/His 469 and Arg 1052/His 1057) highlighted as red stick model. D) Close-up view of the pre-crRNA-processing site, with Arg 1085, Ans 1089 and His 1113 essential for pre-crRNA cleavage (see below) highlighted in pink. The crRNA is shown as orange stick-model.

\section{Recognition of the crRNA by RcCas13a}

The overall common crRNA structure of the binary Cas13a-crRNA complexes consists of a stemloop, a 2 nt bulge at the 3' stem and an A-U base pair at the bottom of the stem, implying a shared shape read-out mechanism of crRNA recognition by this endonuclease family, combined with some sequence specific base-readout. RcCas13a recognizes its $~ 58$ nt long crRNA predominantly via 
shape-recognition, but makes nucleobase-specific interactions with its stem-loop and 3' flank of the crRNA. The peptide backbone in the helical I (Hel 1) domain (resi 276-279) contacts the exocyclic carbonyl group in C-17 and the Hoogsteen-face of G-16 in the loop. The $\varepsilon$-amino group of Lys 179 interacts with ${ }^{7} \mathrm{~N}$ of $\mathrm{A}-15$, and Asp 293 contacts the exocyclic amino group in A-21 (Fig. 2A+B). In addition, a base-specific readout of the bulge formed by A-8 and C-7 at the 3' base of the stem-loop occurs via bi-dental hydrogen-bonding of Arg 23 and the peptide-backbone of the $\beta$-hairpin in the NTD to the Watson-Crick-Franklin face of C-7. (Fig. 2D) The exocyclic amine in A-8 is interacting with the carbonyl oxygen of Ser 684 located in $\alpha$-helix of the Hel 2 domain. Moreover, G-5 and C-1 in the 3' flank of the crRNA form interactions with Arg 695 (Hel 2) and His 788 (HEPN1-2) (Fig. 2D). All other observed interactions of RcCas13a and its crRNA occur in a nucleobase-unspecific manner and the phosphodiester backbone of the crRNA (Supporting Fig. S9+10). Distinct to the crRNAs from Lba, Lbu, Lse and LshCas13a, the stem-loop in the crRNA of RcCas13a is extended by one C-G base pair and an additional $3.4 \AA$ interaction between the twisted C-28 $\left({ }^{3} \mathrm{~N}\right)$ and $\mathrm{G}-5\left({ }^{1} \mathrm{~N}\right)$ at the base of the stem, next to the conserved U-A base-pair can be observed (Supporting Fig. S10). Moreover, RcCas13a does not form any sequence specific contacts with the 5' flank of the crRNA. In contrast, LbuCas13a and LseCas13a interact base-specific with an A and a G, respectively, in the 5' flank. It was shown for LbuCas13a that it is sensitive to mutation in the 5' flank of the crRNA as well as to mutations that impact on the overall shape of the stem loop $(3,10)$. Nucleobases at positions 10-14 in the crRNA spacer region are solvent exposed and are presumably acting as the seed region for target RNA binding (Fig 1B), as shown for the homolog LbuCas13a. (19) The 2-nt bulge at the base of the 3' stem-loop, a common characteristic of so far investigated Cas13a endonucleases (Supporting Fig. S10), was shown to be important to regulate LseCas13a and LshCas13a activity. Here the extended sequence complementarity and base pairing between the crRNA and target-RNA by 8 nucleotides (= anti-tag) blocks activation of the endonuclease. This so called tag:anti-tag interaction appears to allow discrimination between self and non-self, preventing erroneous activation of the endonuclease. $(20,21)$ A consequence of the extended A-form dsRNA formed between crRNA and target-RNA parts of the hairpin and the bulge of the crRNA disintegrate, leading to a misalignment of HEPN 1+2- domain catalytic residues and inactive enzyme. (21)

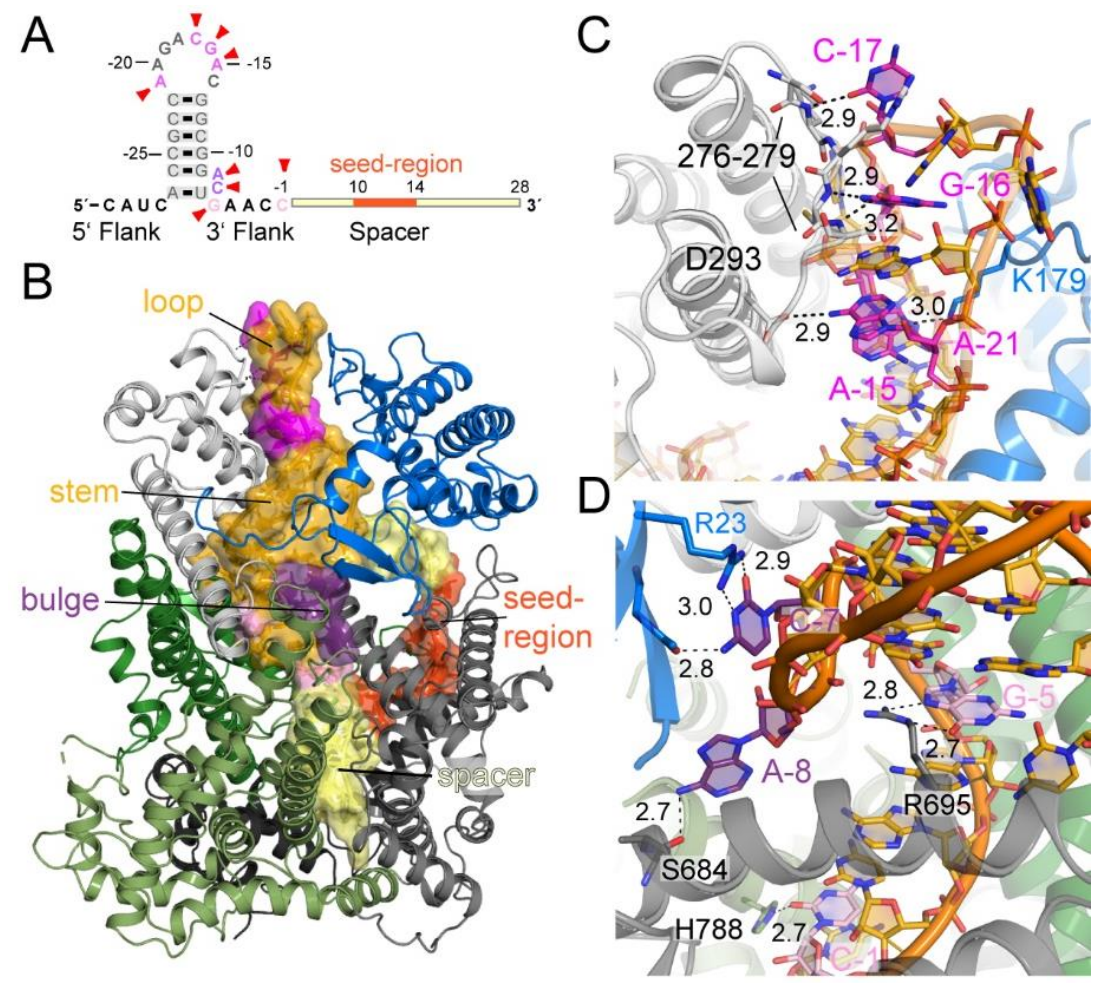

Fig. 2. RcCas13a-crRNA interactions. A) Schematic depiction of the crRNA, with base-specific contacts indicated by red arrows. B) Overall RcCas13acomplex with the crRNA overlaid with its semi-transparent surface. RcCas $13 a$ is colored according to its domain as in Fig. 1. cRNA: stem-loop =golden; spacer = light yellow; seed region = orange; sites with basespecific interaction are highlighted in pink and violet. C) RcCas13a base specific interaction with the tip of the crRNA hairpin, D) with the crRNA bulge at the base of stem and $\mathrm{C}-1$ in the 3' flank. 


\section{Mechanism of pre-crRNA processing}

For some Cas endonucleases from the Cas13a and Cas12a/Cpf1-families, it was shown that they process their own pre-crRNA in a distinct active site $(4,8,22)$, while Cas9-homologs rely on RNaselll, a general RNA processing factor in bacteria, for crRNA biogenesis. (23) We investigated the crRNA processing activity of RcCas13 in vitro, using synthetic 3' 6-Carboxyfluorescein (6-FAM) labelled precrRNA. Our experiments show that RcCas13a does not rely on the catalytic HEPN-domains for precrRNA-processing since RcCas13a mutants, where the active-site residues in one or both of the HEPN1 or HEPN2 domains were mutated to Ala were not affected in their pre-crRNA processing activity (Fig. 3A). Moreover, once bound to RcCas13, the crRNA is not released from the protein again since the binary complex does not cleave 6-FAM-labelled pre-crRNA (Fig. 3A). Furthermore, pre-crRNA processing by RcCas13a is independent of metal ions and the enzyme is still active in the presence of the chelator EDTA in the reaction buffer (Fig. 3B). This indicates an acid-base-catalyzed processing mechanism, in agreement with previous studies on some Cas13a-homologs $(4,8)$ and Cas12a/Cpf1 (22). In order to gain deeper insights into the pre-crRNA processing mechanism of RcCas $13 a$, we mutated all likely candidate amino acid residues located in the vicinity of the 5 ' end of the crRNA in the binary complex structure, since sequence conservation in this area is limited. Following expression and purification, the integrity of all purified proteins was analyzed by dynamic light scattering (DLS) to exclude that the introduced mutations have an impact on protein stability and hence catalytic activity, rather than the chemical properties of the respective amino-acid side chains (Supporting Fig. 11).

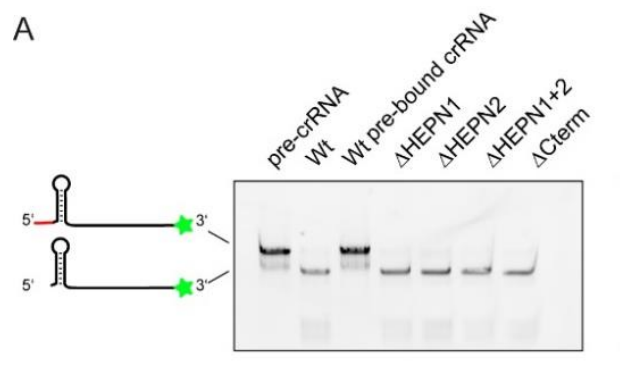

$\mathrm{D}$

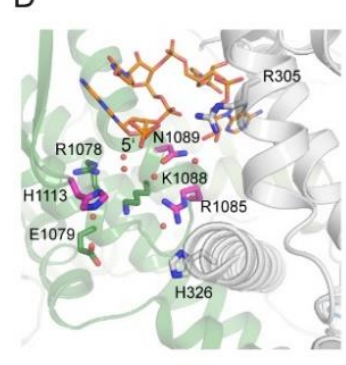

B

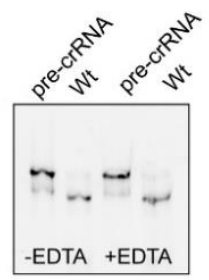

$E$
C

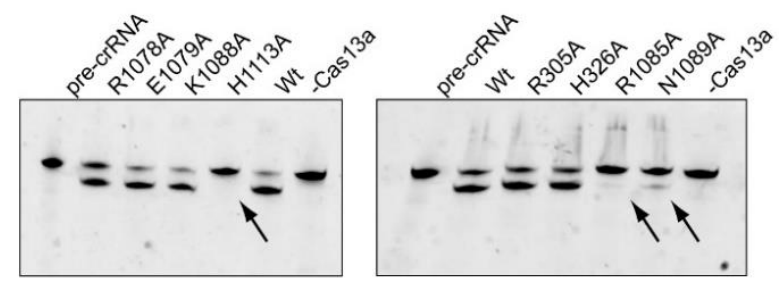

$\mathrm{F}$
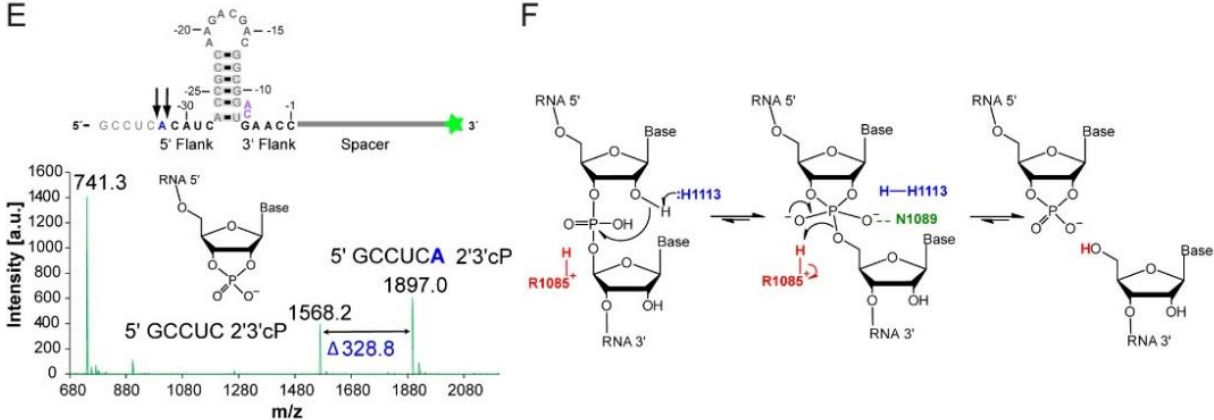

Fig. 3. Processing of pre-crRNA by RcCas13a. A) RcCas13a cleaves 3'-FAM-labelled pre-crRNA independent of its catalytic HEPN-domains or the C-terminal domain. RcCas13a pre-bound to crRNA does not process 3'FAM-labelled pre-crRNA. B) Pre-crRNA cleavage is not inhibited by EDTA and hence metal-ion-independent. C) Identification of residues involved in pre-crRNA processing by mutational analysis. D) Close-up view of the 5 ' end of the crRNA in the RcCas13a binary structure. Residues in the vicinity of the 5' end of the crRNA and subject to mutational analysis are shown as stick model, with the three residues crucial for pre-crRNA processing highlighted in pink. Water molecules are shown as spheres. E) MALDI-TOF analysis of the precrRNA processing reaction shows cleavage of the pre-crRNA 4 or 5 nt 5' of the stem loop, resulting in a 2', 3'cyclic 3' phosphate (2'3'-cP). F) Proposed mechanism of pre-crRNA processing by acid-base catalysis. His 1113 acts as the base, abstracting the proton of the $2{ }^{\prime} \mathrm{OH}$ next to the scissile phosphate. Following a nucleophilic attack by the 2'O the penta-covalent intermediate is stabilized by Asn 1089. Arg 1085 acts as a general acid and protonates the 5'-oxygen leaving group. 
We tested all RcCas13a mutants for their pre-crRNA cleavage activity and could identify Arg 1085, Asn 1089 and His 1113 as key residues essential for pre-crRNA processing. Mutation of these residues to Ala completely or, in case of Asn 1089 partially, abrogates pre-crRNA processing activity (Fig. 3C). While Arg 1085 and Asn 1089 are located at the N-terminus of an a-helix in HEPN2 at the bottom of a cleft between the Hel 1 and HEPN2-domain, His 1113 sits at the tip of a $\beta$-hairpin in the HEPN2-domain (Fig. 3D). While in LbuCas13a Arg 1079, Lys 1082 and Asn 1083 residues at structurally equivalent positions at the $\mathrm{N}$-terminus of the $\alpha$-helix where shown to be crucial in LbuCas13a for pre-crRNA cleavage (8), mutation of the corresponding Lys 1088 in RcCas13a did not impact on its pre-crRNA-processing activity (Fig. 3C, Supporting Fig. 12). In contrast, in the homolog from L. shahii (LshCas13a) an Arg and His located in the NTD are responsible for pre-crRNA cleavage (4). Although all Cas13a homologs whose structures have been determined share the same overall domain structure, they vary in the relative orientation of the domains as well as the exact positioning of the crRNA (Supporting Fig. 6). In general, metal-free acid-base catalysis of nucleases are $\mathrm{S}_{\mathrm{N}} 2$ reactions. Here a nucleophile attacks the scissile phosphate either from the $3^{\prime}$ side, breaking the $5^{\prime}$ O-P bond, which produces 3'-phosphate and 5'-OH, or the 5' side, breaking the 3' O-P bond, resulting in a 5'-phosphate and 3'-OH. (24) We analyzed the reaction products of the in vitro pre-crRNA processing reaction by MALDI-TOF (Matrix Assisted Laser Desorption lonisation- Time Of Flight) mass spectrometry, which reveals that RcCas13a cleaves either 4 or $5 \mathrm{nt} 5^{\prime}$ to the stem loop, generating a 2',3'-cyclic 3' phosphate (2'3' cP, Fig. 3E, Supporting Fig. 13). Given that the reaction product is a 2', $3^{\prime}-\mathrm{cP}$, the 2'-oxygen next to the scissile phosphate has to carry out the nucleophilic attack and is thus transferred to the 5' phosphate. Prerequisite for this transfer reaction is deprotonation of the 2'OH group, most likely by His 1113 acting as the base. However, it is also possible that His 1113 abstracts a proton from a water molecule and that the resulting hydroxide ion acts as the effective base, since our structure shows RcCas13a in complex with the processed, mature crRNA and not the pre-crRNA bound state. The penta-covalent reaction intermediate is then most likely stabilized by Asn 1089, followed by protonation of the 5'-oxygen leaving group by Arg 1085. (Fig. 3F) Due to the high intrinsic $p K a$ value of the Arginine's guanidinium group (pKa 13.8 \pm 0.1 ) and delocalization of the positive charge over many atoms involved in a conjugated m-system, the arginine side chain has the unusual ability to retain its charge under all physiological conditions and can thus function as an acid catalyst. $(25,26)$

\section{In vivo activity of RcCas13a}

We investigated the in vivo cleavage activity of RcCas13a in E. coli JM109 using plasmid encoded reporter genes (luciferase: luxABCDE; green fluorescence protein: GFP) under control of an anhydrotetracycline $\mathrm{P}_{\mathrm{TET}}$ and an IPTG inducible T7-promoter, respectively. RcCas13a and a crRNA with complementary spacer sequence to luxA are under transcriptional regulation of the arabinose responsive $\mathrm{P}_{\mathrm{BAD}}$-promoter (Fig. 4A). This setup allows the distinct expression of all assay components: RcCas13a (wt or mutants), crRNA ${ }^{\text {luxA }}$, luciferase (reporter for cis-cleavage activity), gfp (reporter for trans cleavage activity). Upon expression of wt RcCas13a and crRNA we observe a reduction in bioluminescence due to the sequence specific degradation of luxABCDE mRNA by RcCas13a (Fig. 4B, left red bar, "cis cleavage"), which is independent of expression induction of the second reporter gfp (Supporting Fig. 14). This observed reduction in bioluminescence is strictly dependent on the presence and expression of the luxA-specific crRNA (Fig. 4B, yellow and pink bars). Deletion of the CTD in Cas13a does not affect RcCas13a activity (Fig. 4B, brown bar), while mutation of any of the catalytic residues in the HEPN- domains abolishes RcCas13a activity (Fig. 4B, blue bars). Sequence independent trans-cleavage was analyzed by GFP-fluorescence. Wt RcCas13a and RcCas $13 a \triangle C T D$ reduce GFP-fluorescence only when co-expressed with crRNA and luxABCDE (Fig. 4C, red and brown hatched bars). Without co-expression of either crRNA or the lux-operon GFP- 
A
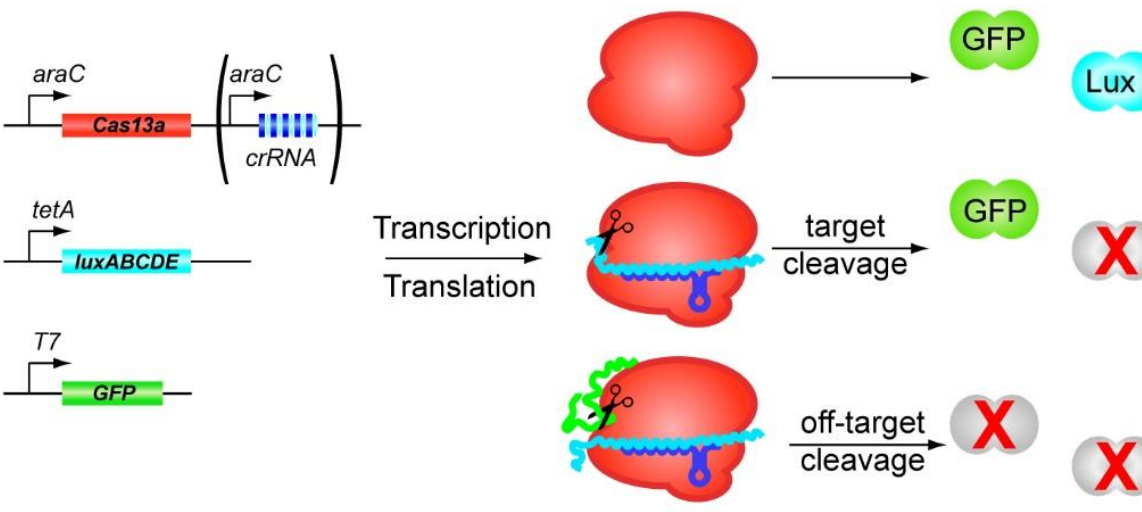

B

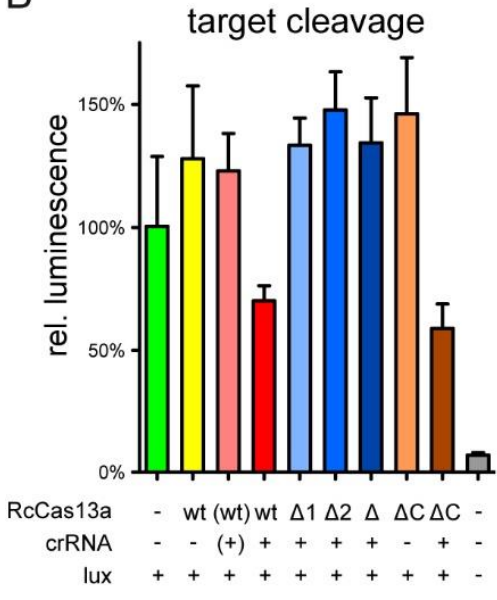

C

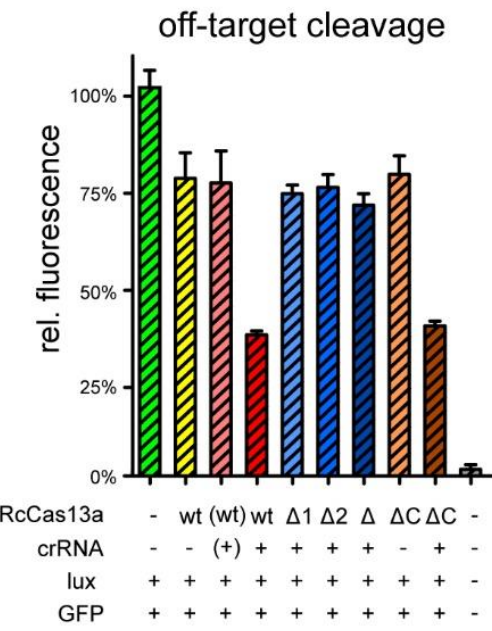

Figure 4: In vivo activity of RcCas13a. A) Schematic depiction of the in vivo activity assay. Two reporter gene constructs (luxABCDE and GFP) as well as expression constructs for RcCas13a variants and crRNA encoded within a CRISPR-locus targeting luXABCDE are introduced into $E$. coli cells. Upon expression of RcCas $13 a$ and $\mathrm{CrRNA}^{\prime \prime x A}$ cis-cleavage activity can be monitored by reduction of luminescence, while sequence unspecific transcleavage can be assessed by alteration in GFP-fluorescence. B) Sequence-specific target cleavage activity (ciscleavage) by RcCas $13 a$ is followed by bioluminescence upon luciferase expression reduction. Wt RcCas $13 a$ and RcCas 13a $\triangle$ CTD reduce bioluminescence in a crRNA/uxA dependent manner (red and brown bar), while all HEPN-mutants lost their impact on luciferase expression (blue bars). C) Sequence-unspecific RNA cleavage (trans-cleavage) was analyzed by measuring GFP-fluorescence upon induction of crRNA/uxA and target RNA expression (luxABCDE). Wt RcCas13a and RcCas13a $\triangle$ CTD reduce GFP-fluorescence only upon expression of $\mathrm{crRNA}^{1 / \mathrm{ux}}$ and target sequence (red and brown hatched bars). Mutation of any of the catalytic residues in the HEPN-domains leads to an inactive RcCas13a (blue hatched bars). RcCas13a and crRNA expression is induced by addition of arabinose to the growth medium. Luciferase and GFP expression is controlled by anhydrotetracycline and IPTG, respectively. (+): Encoding gene/expression construct is present in the $E$. coli cells, but was not induced. (-) empty vector control. $(\Delta 1=\Delta \operatorname{HEPN1}, \Delta 2=\Delta \mathrm{HEPN} 2, \Delta=\Delta \mathrm{HEPN} 1+2 ; \Delta \mathrm{C}=$ $\triangle$ CTD)

fluorescence remained unaffected by RcCas13a expression. (Fig. 4C, yellow hatched bar and Supporting Fig. S14). Again RcCas13a-mediated reduction in GFP-fluorescence is dependent on the HEPN-domains and prevented by mutation of any of the active site residues (Fig. $\mathbf{4 C}$, blue hatched bars). This HEPN-dependent cis- and trans-cleavage activity by RcCas $13 \mathrm{a}$ is common to the Cas13a-family (3-5,7,8,10). Moreover, expression of wt RcCas13a (or RcCas13a $\Delta C T D$ ) in combination with crRNA and target RNA leads to a reduced bacterial growth of the $E$. coli cells (Supporting Fig. S15). This is in agreement with previous studies on Type VI CRISPR-Cas systems, showing that Cas13a mediates protection against phages by dual function: first, by direct target RNA degradation and secondly, abortion of the infectious cycle by inducing growth arrest (dormancy) in the host cell through trans-cleavage of transcripts. With this indirect targeting mechanism Type VI 
systems also act on CRISPR-resistant phages, deplete the phage population and thus provide herd immunity to uninfected bacteria. $(21,27)$

\section{Conclusion}

The photo-auxotrophic bacterium $R$. capsulatus encodes, next to Class 1 , also a Class 2 Type VI CRISPR-Cas system, harboring a $145 \mathrm{kDa}$ Cas13a single-effector endonuclease. This RcCas13a possess two distinct active sites for crRNA processing and ssRNA endonuclease activity that are typical for this family. The X-ray crystal structure of RcCas13a in complex with its crRNA reveals, that the enzyme combines shape and sequence specific readout to recognize its crRNA and shows the enzyme in its stable and contracted, inactive conformation, which likely prevents premature activation of the endonuclease activity. Moreover, RcCas13a processes its crRNA, by cleaving either 4 or $5 \mathrm{nt}$ upstream of the stem loop, which results in a 2'3' cyclic phosphate at the 5' leaving group. Crucial for the crRNA processing activity are three residues located in the processing site: His 1113 and Arg 1085 likely acting as acid and base, respectively, and Asn 1089 stabilizing the intermediate reaction state. Upon expression in E. coli, RcCas13a utilizes its HEPN-domain composite active site to cleave target RNAs with complementary sequence to the crRNA-spacer sequence. The presence of crRNA and complementary target RNA also unlocks the HEPN-domain dependent trans-cleavage activity of bystander RNAs and results in a reduced bacterial growth, indicating that RcCas13a protects the bacterial population by aborting lytic infection and phage propagation by inducing dormancy in the host, as reported for homologs. In summary, our data provide further insights into the structure, mechanism and function of this intriguing family of RNA dependent endonucleases.

\section{Materials and Methods}

\section{Molecular Biology}

Used plasmids and primers are listed in Table S4 and S5. Mutants were prepared using standard cloning procedures (restriction enzymes (New England Biolabs), Gibson Assembly, QuikChange® Mutagenesis (Agilent), Q5 site-directed mutagenesis (NEB), SLIM PCR(28)) according to the protocol of manufacturer's instructions. All used plasmids were verified by Sanger sequencing (GATC Biotech / Genewiz, Germany).

\section{Protein expression of RcCas13a and RcCas13a mutants}

For protein expression RcCas13, annotated as a hypothetical protein (gene bank accession code WP_013067728.1), or RcCas13a mutants were cloned into the respect expression plasmid (see Table S4) and expressed in E. coli Rosetta2 (DE3). Cells obtained from an overnight culture (LB medium supplemented with respective antibiotics according to Table S4, $37^{\circ} \mathrm{C}, 180 \mathrm{rpm}$ ) were diluted 1:100 (OD 600 between $0.05-0.07)$ in $2 Y \mathrm{~T}$ medium (50 $\mathrm{\mu g} / \mathrm{ml}$ kanamycin) and incubated at $37^{\circ} \mathrm{C}, 180$ rpm until an $\mathrm{OD}_{600}$ of 0.7 was reached. After shock-cooling for 15 - 30min on ice, protein expression was induced with $1 \mathrm{mM}$ isopropyl $\beta$-D-1-thiogalactopyranoside (IPTG) and incubated overnight at 18$20^{\circ} \mathrm{C}, 180 \mathrm{rpm}$. Cells were subsequently harvested by centrifugation $\left(5000 \times \mathrm{g} ; 10 \mathrm{~min} ; 4^{\circ} \mathrm{C}\right)$.

\section{RcCas13a and RcCas13a-crRNA complex purification}

All purification steps were performed at $4-8^{\circ} \mathrm{C}$ or on ice unless otherwise noted. The two-step purification procedure of RcCas 13 a proteins comprised a $\mathrm{Ni}^{2+}$-affinity chromatography followed by removal of the $\mathrm{His}_{6}-\mathrm{MBP}$-tag using Tobacco Etch Virus (TEV) protease and a size-exclusion chromatography (SEC), both carried out on an ÄKTA-FPLC system (GE Healthcare, now Cytiva). The cell pellet obtained from a 1-liter expression was resuspended in $30 \mathrm{~mL}$ His-wash buffer (50 mM 4- 
(2-hydroxyethyl)-1-piperazineethanesulfonic acid (Hepes) $\mathrm{pH} 8.0,1 \mathrm{M} \mathrm{NaCl}, 10 \%$ (v/v) glycerol, $1 \mathrm{mM}$ dithiothreitol (DTT), $10 \mathrm{mM}$ imidazole) supplemented with $0.1 \mathrm{mg} / \mathrm{mL}$ DNase I (AppliChem), 0.1 $\mathrm{mg} / \mathrm{mL}$ lysozyme ( 20,000 U/mg; Carl Roth) and one tablet cOmplete ${ }^{\mathrm{TM}}$ ULTRA EDTA-free protease inhibitor tablet (Roche). Cells were lysed by homogenization using an EmulsiFlexC5 (Avestin Inc.) or by sonication and cleared lysate was obtained by centrifugation $\left(24,446 \times \mathrm{g} ; 30 \mathrm{~min} ; 4^{\circ} \mathrm{C}\right)$ and filtration using a Whatman ${ }^{\mathrm{TM}}$ folded filter (GE Healthcare, now Cytiva).

Affinity purification used a $1 \mathrm{~mL}$-HisTrap FF crude column (GE Healthcare, now Cytiva) at a flow rate of $1 \mathrm{~mL} / \mathrm{min}$. After sample application the column was washed with $12 \mathrm{CV}$ His-wash buffer followed by $5 \mathrm{CV}$ of His-wash buffer supplemented with imidazole to a final concentration of $30 \mathrm{mM}$. RcCas13a proteins were eluted with $13 \mathrm{CV}$ His-elution buffer $(50 \mathrm{mM}$ Hepes $\mathrm{pH}$ 8.0, $500 \mathrm{mM} \mathrm{NaCl}, 10 \%(\mathrm{v} / \mathrm{v})$ glycerol, $1 \mathrm{mM}$ DTT, $300 \mathrm{mM}$ imidazole) and collected in $0.5 \mathrm{~mL}$ fractions. Fractions containing the protein were pooled and concentrated with an Amicon Ultracell Centrifugal filter unit (MWCO $50 \mathrm{kDa}$, Merck Millipore) to a volume of $500 \mu \mathrm{L}$ and digested with TEV protease for 2 hours at $8^{\circ} \mathrm{C}$. After centrifugation (16 $000 \times \mathrm{g} ; 10 \mathrm{~min} ; 4^{\circ} \mathrm{C}$ ) the sample was applied onto a HiLoad Superdex 200 (16/60) column (GE Healthcare, now Cytiva). Separation was performed at a flow rate of $0.8 \mathrm{~mL} / \mathrm{min}$ in SEC buffer (50 mM Hepes pH 8.0, $500 \mathrm{mM} \mathrm{NaCl}, 10 \%$ (v/v) glycerol, $1 \mathrm{mM}$ DTT) and $1.0 \mathrm{~mL}$ fractions were collected. Fractions containing the respective monomeric RcCas13a proteins were pooled, concentrated, and either directly used for crystallization trials or flash-frozen in liquid nitrogen and stored at $-80^{\circ} \mathrm{C}$. The RcCas $13-c r R N A$ binary complex was obtained by co-expressing RcCas $13 a$ containing a C-terminal His 6 -tag with the CRISPR-locus encoded on the same plasmid (pET24RcCas13a+crRNA). The co-purification of the RcCas13a-crRNA complex was done analogously to the RcCas13a protein, without removal of the His-tag.

\section{Expression and purification of the SeMet-labeled RcCas13a-crRNA binary complex}

For production of SeMet-labeled protein, was carried out according to the modified protocol by van den Ent and Löwe (29). In short, a culture of E. coli Rosetta2 (DE3) was grown in M9 minimal medium at $37^{\circ} \mathrm{C}$ under agitation until an $\mathrm{OD}_{600}$ of 0.6 was reached, where an amino acids mix (final concentrations: $100 \mathrm{mg} / \mathrm{L}$-lysine, threonine and phenylalanine, $50 \mathrm{mg} / \mathrm{L}$-leucine, isoleucine, valine and L-selenomethionine) was added as solids and incubated for $15 \mathrm{~min}$. Cells were shock-cooled for $30 \mathrm{~min}$ at $4{ }^{\circ} \mathrm{C}$ and protein expression was induced with $1 \mathrm{mM}$ IPTG overnight. Purification was performed as for the unlabeled protein, but using $5 \mathrm{mM} \beta$-mercaptoethanol (IMAC) and $5 \mathrm{mM}$ DTT (SEC) in the respective buffers.

\section{Pre-crRNA cleavage by RcCas13a}

For the in vitro cleavage assay $0.625 \mu \mathrm{M}$ or $0.208 \mu \mathrm{M}$ 3'-FAM-labeled pre-crRNA (Ella Biotech; sequence in Table S3) was incubated with $2 \mu \mathrm{M}$ RcCas13a in cleavage buffer (20 mM Hepes pH 8.0, $100 \mathrm{mM} \mathrm{NaCl}, 50 \mathrm{mM} \mathrm{KCl}, 5 \%(\mathrm{v} / \mathrm{v})$ glycerol, $0.8 \mu \mathrm{g} / \mathrm{ml} \mathrm{BSA}$ ) for $60-95 \mathrm{~min}$ at $37^{\circ} \mathrm{C}$ in the absence or presence of $2 \mathrm{mM}$ EDTA. Reactions were quenched by the addition of Urea loading buffer (New England Biolabs) and incubation at $75{ }^{\circ} \mathrm{C}$ for $15 \mathrm{~min}$ followed by analysis using denaturing polyacrylamide gel electrophoresis (PAGE, $20 \% 19: 1$ acrylamid : bisacrylamid, 8\% (w/v) urea, Trisboric acid-EDTA (TBE), Carl Roth at $200 \mathrm{~V}$ ). In gel fluorescence was analyzed with a GE Healthcare Amersham Al680 Imager.

\section{MALDI-TOF analysis of Cas13a pre-crRNA processing products}

Processed pre-crRNA samples for MALDI experiments were obtained by incubation of 3'-FAMlabeled pre-crRNA (24 $\mu \mathrm{M} ; 72$ pmol) with RcCas13a $(33 \mu \mathrm{M})$ in cleavage buffer for $3 \mathrm{~h}$ at $37^{\circ} \mathrm{C}$. The Cas 13a protein was removed by heat denaturation $\left(95^{\circ} \mathrm{C} ; 10 \mathrm{~min}\right)$ followed by centrifugation $(16000$ $\left.\mathrm{x} \mathrm{g} ; 10 \mathrm{~min} ; 4^{\circ} \mathrm{C}\right)$. Unprocessed pre-crRNA $\left(100 \mu \mathrm{M}\right.$ in $\left.\mathrm{H}_{2} \mathrm{O}\right)$ served as control. MALDI-TOF mass 
spectra were recorded on a Bruker Autoflex II. For the measurements, the samples were desalted against $\mathrm{ddH}_{2} \mathrm{O}$ using a $0.025 \mu \mathrm{m}$ VSWP membrane filter (Merck Millipore) and then co-crystallized with a matrix solution (0.7 M 3-hydroxypicolinic acid, $0.07 \mathrm{M}$ diammonium citrate in 1:1 $\mathrm{H}_{2} \mathrm{O} / \mathrm{MeCN}$ ).

\section{In vivo activity assay}

The sequences encoding the luciferase subunits $\operatorname{lu} x A, \operatorname{lu} x B$, IuxC, IuxD and IuxE from Photorhabdus luminescens (Uniprot IDs P23146, P19840, P23113, P23148, P19482) were cloned into pASK-IBA5 (IBA Lifesciences), under control of an anhydrotetracycline (aTc) inducible promoter. The gene encoding RcCas13a was cloned alone or together with CRISPR loci targeting the luxABCDE genes (sequence in Table S3) into PBAD RSF1031K, under control of the L-arabinose (ara) inducible pBAD promoter. In order to analyze off-target RNA degradation the sequence encoding eGFP in fusion with a C-terminal ssrA degradation tag was cloned into pACYC, inducible with IPTG. Plasmids according to Table S4 were transformed into E. coli JM109 (DE3) (NEB). Freshly prepared chemically defined EZ medium (Teknova), using $2 \%(\mathrm{v} / \mathrm{v})$ glycerol as nutrient source (instead of glucose) and supplemented with the respective antibiotics, was inoculated from overnight cultures in triplicates to an $\mathrm{OD}_{600}$ of 0.2 . Cells were grown in a transparent 96 -well plate (Greiner bio-one) with lid at $30^{\circ} \mathrm{C}$ under agitation until an $\mathrm{OD}_{600}$ of 0.55 was reached. RcCas13a expression was induced with a final concentration of $0.1 \%(\mathrm{w} / \mathrm{v}) \mathrm{L}$-arabinose. Cells were further grown until the $\mathrm{OD}_{600}$ reached 0.7 and the expression of the reporter genes were induced with a final concentration of $2 \mathrm{mg} / \mathrm{L}$ aTc and $0.5 \mathrm{mM}$ IPTG, respectively. Cell growth, the luminescence and the fluorescence were monitored for $24 \mathrm{~h}$ at $30^{\circ} \mathrm{C}$ under agitation in a Spark $10 \mathrm{M}$ plate reader (Tecan).

\section{Protein crystallization and structure determination}

Crystals of the RcCas13a-crRNA binary complex were obtained by mixing 1 volume of protein $(12 \mathrm{mg} / \mathrm{ml})$ and 2 volumes of precipitant $100 \mathrm{mM} 2-(\mathrm{N}$-morpholino)ethanesulfonic acid (MES) pH 6.5 and $25 \%(\mathrm{w} / \mathrm{v})$ polyethylenglycol 6000 (PEG6000) at $20^{\circ} \mathrm{C}$. Crystals of the SeMet-labeled RcCas13a:crRNA binary complex were obtained by mixing 1 volume of protein $(7.5 \mathrm{mg} / \mathrm{ml})$ and 2 volumes of precipitant ( $100 \mathrm{mM}$ MES pH 6.0, $200 \mathrm{mM} \mathrm{NaCl}$ and $20 \%$ (w/v) PEG6000) at $20^{\circ} \mathrm{C}$. Crystals were harvested using nylon loops (Hampton Research), soaked in artificial mother liquor supplemented with $30 \%(\mathrm{w} / \mathrm{v})$ ethylene glycol as cryo-protectant and flash-cooled in liquid nitrogen. X-ray diffraction data were collected at beamlines PX I - X06SA of the Swiss Light Source (SLS) in Villigen, Switzerland, and ID30A-1 (MASSIF-1) of the European Synchrotron Radiation Facility (ESRF) in Grenoble, France. Samples were cooled during data collection at $100 \mathrm{~K}$ with liquid nitrogen. Data collection, data processing and structure refinement statistics can be found in Table S1. Data were processed with XDS (30) and phasing was performed by SAD with $\operatorname{HKL2MAP}(31,32)$ and CRANK2 (33). Manual building of the model was done in COOT (34), refinement was carried out with PHENIX (35) and PDB_Redo (36), and structural figures were prepared with PyMOL (37). The final model was deposited at the Protein Data Bank (PDB) with the accession code 7050.

\section{Analysis of Cas13a by Dynamic Light Scattering (DLS)}

Purified Cas13a-protein samples were analyzed by DLS. DLS measurements were performed using a DynaPro ${ }^{\mathrm{TM}}$ Nanostar $^{\mathrm{TM}}$ dynamic light scattering system (Wyatt Technology, Santa Barbara, CA) and analysis was done by using the DYNAMICS® software (version 8.0.0.77) provided by the manufacturer. Results from 10 consecutive measurements at $25^{\circ} \mathrm{C}$ (laser wavelength $661.3 \mathrm{~nm}$ ) each with an acquisition time of 5 seconds and a read interval of 1 second were averaged for every protein sample. For the solution viscosity-parameter the viscosity of water at $20^{\circ} \mathrm{C}(1.000 \mathrm{cP})$ was used. 


\section{Statements}

This article contains supporting information. The authors declare that they have no conflicts of interest with the contents of this article.

\section{Data availability}

Structure coordinates and structure factor-files have been deposited in the PDB data bank. (PDB code 7OS0). All other data that support the findings of this study are available from the corresponding author upon request.

\section{Acknowledgements}

This work was financially supported by the Deutsche Forschungsgemeinschaft (SCHN 12735/SPP 2141, SCHN 1273-6; Center for Integrated Protein Science Munich (CIPSM, EXC114) and SFB1309). We thank the European Synchrotron Radiation Facility (ESRF) and the Swiss Light Source (SLS) for beamtime, and the staff of beamlines PX I (SLS), MASSIF-1 and ID23-2 (ESRF) for setting up the beamlines for data collection. We also thank Christopher Scheidler and Philipp Merz for assistance with protein purification for some activity assays.

\section{References}

1. Makarova, K. S., Wolf, Y. I., Iranzo, J., Shmakov, S. A., Alkhnbashi, O. S., Brouns, S. J. J., Charpentier, E., Cheng, D., Haft, D. H., Horvath, P., Moineau, S., Mojica, F. J. M., Scott, D., Shah, S. A., Siksnys, V., Terns, M. P., Venclovas, C., White, M. F., Yakunin, A. F., Yan, W., Zhang, F., Garrett, R. A., Backofen, R., van der Oost, J., Barrangou, R., and Koonin, E. V. (2020) Evolutionary classification of CRISPR-Cas systems: a burst of class 2 and derived variants. Nat. Rev. Microbiol. 18, 67-83

2. Watanabe, S., Cui, B., Kiga, K., Aiba, Y., Tan, X. E., Sato'o, Y., Kawauchi, M., Boonsiri, T., Thitiananpakorn, K., Taki, Y., Li, F. Y., Azam, A. H., Nakada, Y., Sasahara, T., and Cui, L. (2019) Composition and diversity of CRISPR-Cas13a systems in the genus Leptotrichia. Front Microbiol. 10, 2838

3. East-Seletsky, A., O'Connell, M. R., Knight, S. C., Burstein, D., Cate, J. H., Tjian, R., and Doudna, J. A. (2016) Two distinct RNase activities of CRISPR-C2c2 enable guide-RNA processing and RNA detection. Nature 538, 270-273

4. $\quad$ Liu, L., Li, X., Wang, J., Wang, M., Chen, P., Yin, M., Li, J., Sheng, G., and Wang, Y. (2017) Two distant catalytic sites are responsible for C2c2 RNase activities. Cell 168, 121-134

5. Abudayyeh, O. O., Gootenberg, J. S., Konermann, S., Joung, J., Slaymaker, I. M., Cox, D. B., Shmakov, S., Makarova, K. S., Semenova, E., Minakhin, L., Severinov, K., Regev, A., Lander, E. S., Koonin, E. V., and Zhang, F. (2016) C2c2 is a single-component programmable RNAguided RNA-targeting CRISPR effector. Science 353, aaf5573

6. East-Seletsky, A., O'Connell, M. R., Burstein, D., Knott, G. J., and Doudna, J. A. (2017) RNA targeting by functionally orthogonal Type VI-A CRISPR-Cas enzymes. Mol. Cell 66, 373-383

7. Liu, L., Li, X., Ma, J., Li, Z., You, L., Wang, J., Wang, M., Zhang, X., and Wang, Y. (2017) The molecular architecture for RNA-guided RNA cleavage by Cas13a. Cell 170, 714-726

8. Knott, G. J., East-Seletsky, A., Cofsky, J. C., Holton, J. M., Charles, E., O'Connell, M. R., and Doudna, J. A. (2017) Guide-bound structures of an RNA-targeting A-cleaving CRISPRCas13a enzyme. Nat. Struct. Mol. Biol. 24, 825-833

9. Kellner, M. J., Koob, J. G., Gootenberg, J. S., Abudayyeh, O. O., and Zhang, F. (2019) SHERLOCK: nucleic acid detection with CRISPR nucleases. Nat. Protoc. 14, 2986-3012

10. Meeske, A. J., Jia, N., Cassel, A. K., Kozlova, A., Liao, J., Wiedmann, M., Patel, D. J., and Marraffini, L. A. (2020) A phage-encoded anti-CRISPR enables complete evasion of type VIA CRISPR-Cas immunity. Science $369,54-59$

11. Lopatina, A., Tal, N., and Sorek, R. (2020) Abortive infection: bacterial suicide as an Antiviral immune strategy. Annu. Rev. Virol. 7, 371-384 
12. Deveau, H., Barrangou, R., Garneau, J. E., Labonte, J., Fremaux, C., Boyaval, P., Romero, D. A., Horvath, P., and Moineau, S. (2008) Phage response to CRISPR-encoded resistance in Streptococcus thermophilus. J. Bacteriol. 190, 1390-1400

13. Pyenson, N. C., Gayvert, K., Varble, A., Elemento, O., and Marraffini, L. A. (2017) Broad Targeting specificity during bacterial Type III CRISPR-Cas immunity constrains viral escape. Cell Host Microbe. 22, 343-353

14. van Houte, S., Ekroth, A. K., Broniewski, J. M., Chabas, H., Ashby, B., Bondy-Denomy, J., Gandon, S., Boots, M., Paterson, S., Buckling, A., and Westra, E. R. (2016) The diversitygenerating benefits of a prokaryotic adaptive immune system. Nature 532, 385-388

15. Hampton, H. G., Watson, B. N. J., and Fineran, P. C. (2020) The arms race between bacteria and their phage foes. Nature 577, 327-336

16. Koonin, E. V., Makarova, K. S., and Zhang, F. (2017) Diversity, classification and evolution of CRISPR-Cas systems. Curr. Opin. Microbiol. 37, 67-78

17. Zimmermann, L., Stephens, A., Nam, S. Z., Rau, D., Kubler, J., Lozajic, M., Gabler, F., Soding, J., Lupas, A. N., and Alva, V. (2018) A completely reimplemented MPI bioinformatics toolkit with a new HHpred server at its Core. J. Mol. Biol. 430, 2237-2243

18. Amir, M., Kumar, V., Dohare, R., Islam, A., Ahmad, F., and Hassan, M. I. (2018) Sequence, structure and evolutionary analysis of cold shock domain proteins, a member of OB fold family. J. Evol. Biol. 31, 1903-1917

19. Tambe, A., East-Seletsky, A., Knott, G. J., Doudna, J. A., and O'Connell, M. R. (2018) RNA binding and HEPN-nuclease activation are decoupled in CRISPR-Cas13a. Cell Rep. 24, 10251036

20. Meeske, A. J., and Marraffini, L. A. (2018) RNA guide complementarity prevents self-targeting in Type VI CRISPR systems. Mol. Cell. 71, 791-801 e793

21. Wang, B., Zhang, T., Yin, J., Yu, Y., Xu, W., Ding, J., Patel, D. J., and Yang, H. (2021) Structural basis for self-cleavage prevention by tag:anti-tag pairing complementarity in type $\mathrm{VI}$ Cas13 CRISPR systems. Mol. Cell 81, 1100-1115

22. Swarts, D. C., van der Oost, J., and Jinek, M. (2017) Structural basis for guide RNA processing and seed-dependent DNA targeting by CRISPR-Cas12a. Mol. Cell 66, 221-233

23. Deltcheva, E., Chylinski, K., Sharma, C. M., Gonzales, K., Chao, Y., Pirzada, Z. A., Eckert, M. R., Vogel, J., and Charpentier, E. (2011) CRISPR RNA maturation by trans-encoded small RNA and host factor RNase III. Nature 471, 602-607

24. Yang, W. (2011) Nucleases: diversity of structure, function and mechanism. Q. Rev. Biophys. 44, 1-93

25. Fitch, C. A., Platzer, G., Okon, M., Garcia-Moreno, B. E., and McIntosh, L. P. (2015) Arginine: Its pKa value revisited. Protein Sci. 24, 752-761

26. Silva, P. J., Schulz, C., Jahn, D., Jahn, M., and Ramos, M. J. (2010) A tale of two acids: when arginine is a more appropriate acid than $\mathrm{H}_{3} \mathrm{O}^{+}$. J. Phys. Chem. B 114, 8994-9001

27. Meeske, A. J., Nakandakari-Higa, S., and Marraffini, L. A. (2019) Cas13-induced cellular dormancy prevents the rise of CRISPR-resistant bacteriophage. Nature 570, 241-245

28. Chiu, J., March, P. E., Lee, R., and Tillett, D. (2004) Site-directed, ligase-independent mutagenesis (SLIM): a single-tube methodology approaching 100\% efficiency in 4 h. Nucleic Acids Res. 32, e174

29. Van Duyne, G. D., Standaert, R. F., Karplus, P. A., Schreiber, S. L., and Clardy, J. (1993) Atomic structures of the human immunophilin FKBP-12 complexes with FK506 and rapamycin. J. Mol. Biol. 229, 105-124

30. Kabsch, W. (2010) XDS. Acta Crystallogr. D Biol. Crystallogr. 66, 125-132

31. Pape, T., and Schneider, T. R. (2004) HKL2MAP: a graphical user interface for phasing with SHELX programs. J. Appl. Cryst. 34, 843-844

32. Sheldrick, G. M. (2008) A short history of SHELX. Acta Crystallogr. A 64, 112-122

33. Skubak, P., Arac, D., Bowler, M. W., Correia, A. R., Hoelz, A., Larsen, S., Leonard, G. A., McCarthy, A. A., McSweeney, S., Mueller-Dieckmann, C., Otten, H., Salzman, G., and Pannu, N. S. (2018) A new MR-SAD algorithm for the automatic building of protein models from lowresolution X-ray data and a poor starting model. IUCrJ 5, 166-171

34. Emsley, P., Lohkamp, B., Scott, W. G., and Cowtan, K. (2010) Features and development of Coot. Acta Crystallogr. D Biol. Crystallogr. 66, 486-501 
35. Liebschner, D., Afonine, P. V., Baker, M. L., Bunkoczi, G., Chen, V. B., Croll, T. I., Hintze, B., Hung, L. W., Jain, S., McCoy, A. J., Moriarty, N. W., Oeffner, R. D., Poon, B. K., Prisant, M. G., Read, R. J., Richardson, J. S., Richardson, D. C., Sammito, M. D., Sobolev, O. V., Stockwell, D. H., Terwilliger, T. C., Urzhumtsev, A. G., Videau, L. L., Williams, C. J., and Adams, P. D. (2019) Macromolecular structure determination using X-rays, neutrons and electrons: recent developments in Phenix. Acta Crystallogr. D Struct. Biol. 75, 861-877

36. Joosten, R. P., Long, F., Murshudov, G. N., and Perrakis, A. (2014) The PDB_REDO server for macromolecular structure model optimization. IUCrJ. 1, 213-220

37. Schrödinger, L. L. C. (2015) The PyMOL Molecular Graphics System, Version 1.8. 